\title{
Spatial accessibility assessment to healthcare facilities: urban and rural areas
}

\author{
Inna Č́belková ${ }^{1 *}$, Zhanna Gardanova ${ }^{2}$, Eduard Neimatov $^{3}$, and Vladimir Esaulov ${ }^{2}$ \\ ${ }^{1}$ Czech University of Life Sciences Prague, Department of Trade and Finance, Faculty of Economics \\ and Management, Kamýcká 129, Prague 6, 16500 Prague, Czech Republic \\ ${ }^{2}$ Pirogov Russian National Research Medical University, 1 Ostrovitianov str., 117997, Moscow, \\ Russian Federation \\ ${ }^{3}$ I.M. Sechenov First Moscow State Medical University of the Ministry of Health of the Russian \\ Federation (Sechenov University), 2 Bolshaya Pirogovskaya str., Moscow, 119991, Russian Federation
}

\begin{abstract}
This research concentrates on the spatial accessibility assessment to healthcare facilities. Specifically, in doing so it compares the situation in urban and rural areas. In many countries, health inequalities continue to be a major public health challenge. Furthermore, there is also urbanization that occurs when people move from rural areas to urban areas. It leads to an increase in urban population and the extent of urban areas. Driven by population growth, many cities in low and middle-income countries are growing at record rates which puts large pressure on the healthcare system and facilities. Our research demonstrates that due to many political, economic, social and cultural factors, the current inequalities in health care between regions, urban and rural areas and different age and income groups are increasing. Thence, there is a pressing need for the improvement of the healthcare in the lagged regions and areas using such means as the advanced technologies, analysis of data, as well as targeted funding and assistance to those who need it the most.
\end{abstract}

Keywords: spatial accessibility, the gap between urban and rural areas, health care, differentiation.

\section{Introduction}

Inequalities in healthcare cover several dimensions of service delivery, use of health care, health outcomes, reimbursement for health insurance and access, among others [1,2]. A key element that needs to be taken into account when examining geographical disparities at the end of life is how the accessibility of health services is influenced by the distance and the time it takes for people to reach them, as well as by the geographical distribution of healthcare providers and patients who benefit from their services. Locality-related issues have been discussed in health justice research, but most efforts focus on describing and analysing the distribution of social determinants of health. It seems necessary to include in this analysis comparing the total population in a health sector with the population with access to services (palliative care and administrative barriers). In order to determine whether a given area has

* Corresponding author: cabelkova@pef.czu.cz 
inadequate health services, the geographical accessibility of health services refers to the relative access to health services in a given area [3-5]. Spatial accessibility is influenced by distance, travel time and spatial distribution of healthcare providers and consumers. It can be divided into two main categories which have the potential to demonstrate spatial accessibility based on actual use of health services [6]. The geographical accessibility of health services identified refers to the actual use of health services in a given location, while the potential geographical accessibility of health services refers to the totality of health services available in an area. The determinants of accessibility are separated by geographic location when assessing spatial accessibility [7, 8].

Complex access calculations using spatial interaction processes, distance disintegration and the manipulation of demand and supply data at the local level [9]. Based on a review of accessibility measures in literature, Wang and Tang proposed to fine tune the popular 2-step method of floating catchments to take these aspects into account. A multi-criteria decision analysis has been developed to identify optimal locations for new health facilities in disadvantaged areas. An index for the spatial accessibility of health facilities is defined as the disparity between a regional block for the accessibility of health facilities. From the same consideration of the M-habitants and location of the facilities, an accessibility index is measured in such a way that the accessibility index of the inhabitants in the ITH area is the supply capacity of the facility (Th) and hospital demand in the CTH area (Village Population) and the distance between residential areas is the distance of the facility, which decreases depending on the number of residential areas and the number of facilities (indexed residential areas $=1$ and indexed facilities $=1)$ [10].

The spatial accessibility of health facilities has received huge attention in Chinese case studies and county-level studies but the availability of high-resolution geodata is limited. For example, Tao et al. measured the accessibility of elderly people to care facilities in China using census data at the sub-district level to optimise the position of these facilities in order to achieve spatial equality. The results show that public health facilities within the study area were grouped in a distribution with central regions having high density of facilities and peripheral regions having low density. In order to assess this from a transport perspective it is crucial to understand the availability of health facilities and intensive care beds in urban and rural areas [11].

Recent studies on China's health accessibility have highlighted the advantages and benefits of the combined proximity methodology for investigating urban or rural areas. These two methods can be used to identify areas of high and low accessibility across an entire state [12]. This rural focus was driven by the realization that distance is an obvious obstacle in densely populated areas, as well as the alarming decline in the supply of healthcare workers in rural America. The decline in research on the spatial accessibility of cities for healthcare providers corresponds to the increasing availability of powerful software and hardware required for valid and advanced urban studies. One reason is that intuitive spatial indicators suitable for large rural areas have been described as less relevant in congestion-bound urban areas. Dos Anjos Luis and Cabral investigated the geographical accessibility of primary health care facilities in Mozambique using GIS to facilitate healthcare planning [13]. Mansour (2016) examined the accessibility of health facilities in Riyadh, Saudi Arabia [14]. In general, it all confirms that old postulate that distance from health care providers was recognised as a major barrier to accessing health care in the United States since the $19^{\text {th }}$ century [15]. It is important that concrete efforts are made to monitor inequalities in geographical access to healthcare and to achieve the $3^{\text {rd }}$ United Nations Sustainable Development Goal by collecting and analysing relevant open data from the GIS.

To this end it is important to measure access to health facilities in order to identify disadvantaged areas and people. There is a need to connect and compare different accessibility measures, which is why it is suitable for urban planning analyses. In this way, 
politicians and managers can link health services with the valuable information provided by the available data.

\section{Spatial accessibilities of COVID-19 patients to healthcare}

More than a billion people worldwide have little or no access to basic health care, leaving them vulnerable to preventable diseases that can worsen otherwise treatable diseases and cause death. This problem is particularly acute in low and middle-income countries (LMICs) $[16,17]$.

However, the lack of current spatial data and health information complicates the assessment of spatial accessibility and the identification of optimal locations for these facilities. Researchers have also examined inequalities in accessibility between vulnerable groups and other residents [18]. Luo and Whippo proposed a modified two-step method for floating catchments to study the medical availability of older people on various types of roads [19]. A two-step floating catchment methodology has been developed with the thought that people are more likely to visit the nearest hospital than others, to remove the limitations of distance reduction in catchment areas where all residents of the same catchment area are assumed to have the same spatial accessibility. This method was used in our study but when it comes to measuring spatial accessibility, it has limitations. Alternative thresholds were used to assess spatial accessibility such as a study in Scotland which showed that $94 \%$ of the population could get to intensive care units within 45 minutes of driving [20].

There are indications that the inequalities of health resources in China and other countries may explain the high mortality rates in areas with a low number confirmed COVID-19 cases. In Wuhan, researchers found that communities in central urban areas have a high accessibility by car to medical facilities, but access by car in half the communities was inadequate due to their population size [21]. Spatial differences in medical facilities are known to be a decisive reason for uneconomic access and poor health outcomes.

It is essential to understand the availability of health facilities and intensive care beds in urban and rural areas to assess this from a transport perspective. The spatial accessibility index defines the differences among regions in access to health care by block in a region. Accessibility measures consider the proven range of the population as the straight range within a supply network that provides medical services within a time threshold. Changes in accessibility disparities suggest that hospitals with moderate accessibility serve a larger number of residents, while hospitals that provide health services to many residents tend to provide more moderate services. The next step would be to use the best methods to obtain accessibility scores at postcode level to identify the areas with the highest and lowest accessibility to health resources.

An improved measure to improve the accessibility of health services can be done by incorporating the temporal dimension of population requirements across various age groups. Two methods were used to identify areas with high and low access. The results suggest that reduced access to intensive care beds is associated with higher percentage of COVID-19related deaths and the number of cases with greater. At the regional level, it has been found that in regions with less access, the rate of the disease was higher. This could be explained by the higher spatial accessibility if compared to previous studies [22]. Hospitals are urged to use existing response plans and modify them to meet healthcare needs. Value comparisons are relative measures and do not quantify the magnitude of the problem in a specific area of access to health resources [23]. The value is negative when patients have difficulty accessing health resources effectively. 


\section{Low income and black communities have less healthcare access}

It is not that the researchers are not worried about lack of access to resources but that in this case they do not view such failings as affecting all aspects of poverty. One can note that decentralisation of poverty in relation to the rest of the population had a negative impact on access to employment opportunities and facilities for welfare recipients [24]. Although beneficiaries are concentrated in city centres, they have better access to amenities and employment opportunities, but decentralisation has led to a decrease in access to the services. We have reason to believe that the decline of access to jobs is due to changes in employment opportunities for poor households as well as other factors [25, 26]. For example, in the United States, rural Americans who constitute at least $15-20 \%$ of the US population face inequalities that result in poorer health care than urban and suburban residents [27]. According to a 2017 report by the NC-RHRP (North Carolina Rural Health Research Program) at the University of North Carolina at Chapel Hill, rural populations have lower median incomes than urban areas, higher proportion of children living in poverty, fewer adults with postsecondary education, more uninsured residents over 65 years old and higher mortality rates [28]. Researchers identified specific populations most likely to face barriers to health care, including the homeless, low-income people, and migrant workers. Mental health and primary care: Some geographical areas lack adequate access to primary care. Vulnerable populations in areas with poor access to health facilities are more likely to be screened and hospitalized than those with COVID-19 patients [29, 30].

By definition, differences in healthcare outcomes are not differences in health outcomes based on race or ethnicity, but rather disproportionate differences due to variables other than access to care. Given the known risk factors for COVID-19 complications, the confluence of high blood pressure, diabetes, obesity and a higher prevalence of cardiovascular disease in black individuals could trigger some of the early signals [31]. Data on adaptation to comorbidities are not reported, but it is likely that not all or most of the differences in disease rates and outcome can be explained by concomitant comorbidities. The presence of diabetes mellitus, chronic kidney disease and terminal kidney disease predicts a high risk of amputation for black patients with claudication with low SES, similar to that of white patients living in high SES neighbourhoods. The black race has long been shown to be a risk factor for peripheral artery disease (PAD) amputations and race has been argued as a marker of socioeconomic status for true inequality. Black race and low SES still are significant risk factors for amputations from the PAD presentation in the predicted analysis of amputation risk. In patients with peripheral artery disease, white race and low socioeconomic status (SES) were predictors of major amputations [32]. Therefore, it is important to study the effects of racial differences between various ethnic groups and nationalities. There are factors associated with racial and ethnic differences, the lack of an experimental design precludes causal conclusions.

\section{COVID-19 infection and its impact on the demand of hospitalization services}

The models built to predict the impact of COVID-19 infection on healthcare include estimates of the total number of new cases, hospitalizations and admission of patients requiring mechanical ventilation [33]. The data collected at local levels are often used to carry out iterative model revisions. Validating the model projections in order to develop strategies for hospital planning in the event of future epidemics is also required. Projections of hospital occupancy and use of beds and ventilators are based on clinical parameters in COVID-19 patients in the United States by the American Hospital Association and Penn 
Medicine. Researchers developed a model to estimate the appropriate number of COVID-19 patients who would be able to find space in addition to the anticipated non-COVID-19 demand during the pre-pandemic phase. The first component was a state-wide estimate of COVID-19 hospitalizations calculated using data released by the New York City Department of Health and Mental Hygiene on the total number of inpatient COVID-19 cases in the city $[34,35]$. This estimate is scaled to the national estimate using population estimates from the US Census, converted to hospital days and then applied to the four-month period for AHAs modelling the virus' net financial impact on hospitals and health systems. At this point when the number of inpatient COVID-19 cases is declining, hospitals should think about how they can care for patients who need them and plan for possible future increases in COVID-19 cases. The model can be used in the analysis to determine whether reintroduction of elective surgery would allow a change in hospital care without intervention. In order to determine whether the threshold number of patients and capacity requirements of COVID-19 would have been exceeded without intervention, the same model can be used to assess the impact of intervention implementation in combination with the increase in base capacity and patient occupancy. Hospital price growth in the healthcare sector has surpassed the total price growth in 2016 since the pandemic started but the gap has widened since then. In the light of emerging evidence that patient behaviour has changed and those alternative admissions such as home care and remote monitoring are more acceptable to patients, new hospital admissions with lower baseline values could result in the future [36, 37].

In Mexico, the federal government adopted guidelines on hospital restructuring in April 2020 , which aim to expand the capacity of health services by upgrading physical facilities of existing hospitals in higher COVID-19 hospitals. This strategy cost 2 billion Mexican pesos (about $\$ 89.7$ million) to expand MCMA's hospitalization capacity. While hospitals and health systems throughout the country support frontline staff, some hospitals are also located in areas considered hotspots for COVID-19 [38]. A possible shift in explicit demand will have a direct impact on the health of the population, which will mean an increase in cases in a stricter form will have to be covered. In the short and medium term, the possibility of meeting health needs by unallocated emergency services will cause problems. However, the opportunity costs of releasing resources for COVID-19 patient management are likely to decrease due to improper emergencies, which could have a positive impact. Although there is no immediate direct impact of COVID-19 on specific geographical conditions, the temporary effects of deferred care will create new challenges for individuals and the US health care system, increasing the annual cost of the US from $\$ 30$ billion to $\$ 65$ billion in 2017. For every million patients seeking treatment, there is a direct cost to the health care system of $\$ 5.3$ billion [39]. In addition, over 40 percent of respondents to a recent McKinsey Consumer Healthcare Insights survey said that they had cancelled future appointments (for example, routine check-ups or treatments for chronic illnesses), and another 12 percent said they needed care but should not [40]. Thence, the direct impact of COVID-19 on mortality and healthcare costs is significant. Over $90 \%$ of the patients with COVID net identified in the hospital had at least one underlying disease with general well-being (obesity, high blood pressure, chronic lung disease, diabetes mellitus, cardiovascular disease).

Based on published case series from China and other regions, reports on the clinical course of the observed patients during the epidemic, local information on the regional population and the care of three hospitals in our health system, models can be used to model the number and rate of infections in the regional population. The model makes deterministic predictions over time about the number of future infections with specified values for initial disease prevalence, population size, recovery time and transmission rate. This data and simulations can help healthcare and predict the demand for healthcare facilities in rural and urban areas which make them particularly useful. 


\section{Conclusions}

As urbanization progresses and the population of the world grows, urban population will increase by 2.5 billion in 2050, especially in Africa and Asia. Although the world's urban areas are diverse, many cities and municipalities face problems such as unemployment, homelessness, growing squatted settlements, inadequate services and infrastructure, poor health and education services and high pollution rates. Rapid expansion of cities across borders driven by increasing population and infrastructure development has resulted in these cities expanding and swallowing neighbouring urban areas into megacities. These factors lead to both temporary and permanent migration into urban areas. Much of urban migration is driven by rural populations that want the benefits that urban areas offer. Many industries are located in cities and offer opportunities with higher wage levels in urban settings. Urbanization is attractive to many rural dwellers because it offers many opportunities to improve their quality of life. Benefits offered by urban areas include greater opportunities for education, health care and services such as entertainment. Fertility rates in cities are lower in some regions of the world than in rural areas, causing the growth of urban areas. There are many elements of urbanization that a city can turn to against its citizens if it does not plan correctly. Cities have accepted urbanization because of its rapid population growth, but they need to know how to work together, not only to respond to the negative effects of urbanization but also to survive. The current pace of urbanization in developing countries makes urban governance and appropriate planning more urgent.

The relationship between urbanization and development is a major political concern in Africa and Asia. When examining the arguments and evidence for how rapid population growth in cities can increase living standards, the most important results are the development effects of urbanization, the extent of agglomeration, and economic variables. The introduction and growing concentration of the world's population in large cities and cities in low and middle-income countries mean that the relationship between urbanization and development has become a major political concern. Urbanization in low-income countries gives billions of people better access to jobs, health services and the global economy. But, to reap the benefits, nations must face looming health and environmental challenges to urban life. The biggest practical challenges are in Africa and in Asia, where the crucial question is whether rapid urban population growth can help raise living standards, reduce poverty and destroy the ecosystems on which life depends.

For example, in China, quality of healthcare services varies widely between cities and regions. In general, health services in large cities are superior to health services in small and medium towns, and urban health services are superior to rural areas. Urbanization and urban modernization in China have not improved residents' health, because low-income people, particularly low-income and migrant workers, have little access to good health care in large cities, owing to their limited financial resources. Among other things, the spatial distribution opportunities offered by cities have prevented whole neighbourhoods and population groups from accessing adequate health care, good schools, sanitation, tap water, employment and housing. Uncontrolled growth in many cities has led to inadequate provision of public services and the failure to guarantee a minimum quality of life for urban dwellers.

Similar problems affect developing countries where increasing inequality is the result of rapid urbanization trends. Having been driven by the rapid urban growth, the efficiency has led to less equitable urban development. In many cases, both rural and urban migrant workers are attracted to economic opportunities in urban areas by low-skilled and unskilled workers but cannot afford work or housing in urban slums. Some researchers proposed policies to foster labour-intensive growth to absorb the influx of low-skilled or unskilled workers. This contributes to reducing fertility in urban areas and changing the course of overall population growth. The urbanization process is especially pronounced in Africa, Asia and much of the 
world, where population growth occurs. There is an enormous opportunity for sustainability if the right policies are implemented. Urban life has the potential to use resources sustainably more effectively, create sustainable land use and protect biodiversity and natural ecosystems. The growth of urban areas is accompanied by an increasing migration to cities and the flora and fauna of the urban population. The increasing redistribution of Earth's population is likely to impact the planet's natural systems by the interplay of urban environment and population. Much of the population growth that created the world's largest and poorest cities was natural: cities such as Cairo, Delhi, Karachi or Lagos grew because their inhabitants lived longer, fewer of their children died and people migrated to them in search of work. For urbanization to be reversed, many poor cities today must-see urban populations in cities with a strong economic base increase.

All of these demonstrates that in the years to come urban and rural areas around the world (in particular, in the fast-growing cities or in the low-income countries) are going to have growing disparities in the spatial accessibility assessment to healthcare facilities. Governments of the wealthier countries have to think out the strategies how to get rid of these inequalities and to support the access to healthcare for all those who need it. Strategies need to be elaborated and plans need to be made to make this happen for the future of this planet depends on it.

Our results and findings gain particular importance in the times of the COVID-19 pandemic that imposed a large burden on the healthcare systems worldwide. Some of the solutions we propose might be helpful for easing these systems and optimising their access in urban and rural areas

\section{References}

1. X. Zhang, M. Dupre, L. Qiu, W. Zhou, Y. Zhao, D. Gu, BMC Geriatrics, 17(1), 1-11 (2017)

2. C. Willging, D. Sommerfeld, E. Jaramillo, E. Lujan, R. Bly, E. Debenport, R. Lujan, BMC health services research, 18(1), 1-16 (2018)

3. C. Dupin, M. Pinon, K. Jaggi, C. Teixera, A. Sagne, N. Delicado, BMC Nursing, 19(1), $1-5(2020)$

4. J. Lu, C. Zhang, L. Ren, M. Liang, W. Strielkowski, J. Streimikis, International Journal of Environmental Research and Public Health, 17(15), 5265 (2020)

5. P. Apparicio, M. Abdelmajid, M. Riva, R. Shearmur, International Journal of Health Geographics, 7(1), 1-14 (2008)

6. W. Luo, Y. Qi, Health \& Place, 15(4), 1100-1107 (2009)

7. G. Klauss, L. Staub, M. Widmer, A. Busato, BMC health services research, 5(1), 1-15 (2005)

8. L. Mériade, C. Rochette, Social Science \& Medicine, 270, 113658 (2021)

9. B. Surya, D. Ahmad, H. Sakti, H. Sahban, South Sulawesi Province, Indonesia. Land, 9(3), 95 (2020)

10. F. Wang, Q. Tang, Environment and Planning B: Planning and Design, 40(2), 195-212 (2013)

11. Z. Tao, Y. Cheng, J. Liu, International Journal for Equity in Health, 19(1), 1-16 (2020)

12. J. Luo, L. Tian, L. Luo, H. Yi, F. Wang, BioMed research international, 2017, 2094654 (2017)

13. A. Dos Anjos Luis, P. Cabral, P. International Journal for equity in Health, 15(1), 1-13 (2016) 
14. S. Mansour, Geo-spatial Information Science, 19(1), 26-38 (2016)

15. N. Douthit, S. Kiv, T. Dwolatzky, S. Biswas, Public health, 129(6), 611-620 (2015)

16. N. Hennig, Journal of Moral Theology, 1(1), 181-192 (2021)

17. Y. Chen, Y. Assefa, BMC Public Health, 21(1), 1-15 (2021)

18. S. Reshadat, A. Zangeneh, S. Saeidi, R. Teimouri, T. Yigitcanlar, Journal of Public Health, 27(4), 519-529 (2019)

19. W. Luo, T. Whippo, Health \& place, 18(4), 789-795 (2012)

20. J. Bauer, D. Brüggmann, D. Klingelhöfer, W. Maier, L. Schwettmann, D. Weiss, D. Groneberg, Intensive Care Medicine, 46(11), 2026-2034 (2020)

21. S. Chen, W. Su, J. Chen, K. Li, Transformations in Business and Economics, 20(2), 4161 (2021)

22. S. Hamidi, S. Sabouri, R. Ewing, Journal of the American Planning Association, 86(4), 495-509 (2020)

23. P. Austin, J. Merlo, Statistics in Medicine, 36(20), 3257-3277 (2017)

24. M. Niedzielski, E. Eric Boschmann, Annals of the Association of American Geographers, 104(6), 1156-1182 (2014)

25. E. Bordea M. Manea A. Pelligrini, Czech Journal of Social Sciences, Business and Economics, 6(2), 6-14 (2017)

26. I. Cabelkova, W. Strielkowski, Society and Economy, 35(4), 513-529 (2013)

27. K. Walsh, American Political Science Review, 517-532 (2012)

28. Warshaw, R. https://www.aamc.org/news-insights/health-disparities-affect-millionsrural-us-communities (2017)

29. N. Bailey, J. Minton, Urban Geography, 39(6), 892-915 (2018)

30. C. Hochstenbach, S. Musterd, Urban Geography, 39(1), 26-53 (2018)

31. C. Yancy, Jama, 323(19), 1891-1892 (2020)

32. S. Arya, Z. Binney, A. Khakharia, L. Brewster, P. Goodney, R. Patzer, P. Wilson, Journal of the American Heart Association, 7(2), e007425 (2018)

33. M. Meltzer, A. Patel, A. Ajao, S. Nystrom, L. Koonin, Clinical Infectious Diseases, 60(suppl_1), S52-S57 (2015)

34. G. Anesi, J. Jablonski, M. Harhay, J. Atkins, J. Bajaj, C. Baston, J. Gutsche, Annals of Internal Medicine, 174(5), 613-621 (2021)

35. S. Brüggemann, T. Chan, G. Wardi, J. Mandel, J. Fontanesi, R. Bitmead, Informatics in Medicine Unlocked, 100618 (2021)

36. M. Neely, D. Carmichael, American Behavioral Scientist, 00027642211003162 (2021)

37. J. Fang, A. Collins, S. Yao, Journal of Asian Economics, 74, 101300 (2021)

38. Z. Fowler, E. Moeller, L. Roa, I. Castañeda-Alcántara, T. Uribe-Leitz, J. Meara, A. Cervantes-Trejo, PLoS One, 15(11), e0241954 (2020)

39. S. Bartsch, M. Ferguson, J. McKinnell, K. O'Shea, P. Wedlock, S. Siegmund, B. Lee, Health Affairs, 39(6), 927-935 (2020)

40. McKinsey \& Company, https://www.mckinsey.com/industries/healthcare-systems-andservices/our-insights/understanding-the-hidden-costs-of-covid-19s-potential-impacton-us-healthcare\# (2020) 